\title{
Lower Bounds for the Average Genus of a CF-graph
}

\author{
Yichao Chen \\ College of Mathematics and Econometrics \\ Hunan University, Changsha 410082, P.R.China \\ ycchen@hnu.edu.cn
}

Submitted: Nov 15, 2009; Accepted: Oct 28, 2010; Published: Nov 5, 2010

Mathematics Subject Classifications: 05C10

\begin{abstract}
CF-graphs form a class of multigraphs that contains all simple graphs. We prove a lower bound for the average genus of a CF-graph which is a linear function of its Betti number. A lower bound for average genus in terms of the maximum genus and some structure theorems for graphs with a given average genus are also provided.
\end{abstract}

\section{Introduction}

A graph is often denoted by $G=(V, E)$, it is simple if it contains neither multiple edges nor self-loops. If a graph does not contain self-loops but contains multiple edges, we call it a multigraph, otherwise if it contains self-loops, we call it a pseudograph. The graph with only one vertex and no edges is called the trivial graph. The vertex-connectivity $\kappa(G)$ of a graph $G$ is the minimum number of vertices whose removal from $G$ results in a disconnected or trivial graph. The edge-connectivity $\kappa_{1}(G)$ of $G$ is the minimum number of edges whose removal from $G$ results in a disconnected or trivial graph. A spanning tree of $G$ is a tree which is a subgraph of $G$ with the same vertex set as $G$. For a spanning tree of $G$, the number of co-tree edges is called the Betti number of $G$, denoted by $\beta(G)$.

A surface means a compact closed 2-manifold without boundary. It is known that there are two kinds of surfaces, orientable and nonorientable. An embedding of $G$ into a surface $S$ is a topological embedding $i: G \rightarrow S$ (see [14]) and each component of $S-i(G)$, called a region, is homeomorphic to an open disk. In this paper, we only consider embeddings of $G$ into orientable surfaces $S$. A rotation at a vertex $v$ of a graph $G$ is a cyclic order of all edges incident with $v$, thus an $n$-valent vertex admits $(n-1)$ ! rotations. A rotation system $R$ of the graph is a collection of rotations, one for each vertex of $G$. An embedding of $G$ into an orientable surface $S$ induces a rotation system as follows: the rotation at $v$ is the cyclic permutation corresponding to the order in which the edge-ends are traversed in an orientation-preserving tour around $v$. Conversely, by the Heffter-Edmonds principle, every rotation system induces a unique embedding (up to homeomorphism) of $G$ into some 
orientable surface $S$. The bijection of this correspondence implies that the total number of orientable embeddings is $\prod_{v \in G}\left(d_{v}-1\right)$ !.

The average genus $\gamma_{a v g}(G)$ of a graph $G$ is the expected value of the genus random variable, over all labeled 2-cell orientable embeddings of $G$, using the uniform distribution. The investigation of average genus will help us to understand embeddings of graphs better. We also show that it is connected with the mode of embedding distribution sequence [12]. See $[1,3,4,5,6,7,15,20,21]$ etc. for more details.

$A$ cactus is a graph obtained in the following way: start with a tree $T$, then replace some of the vertices in $T$ by simple cycles and connect the edges incident to each such vertex to the corresponding cycle in an arbitrary way. A necklace $N_{r, s}$ of type $(r, s)$ is a cycle where $r$ disjoint edges are doubled and $s$ self-loops are added to $s$ vertices which are not endpoints of doubled edges. Figure 1 shows two necklaces of type $(2,2)$ and a cactus with six vertices.
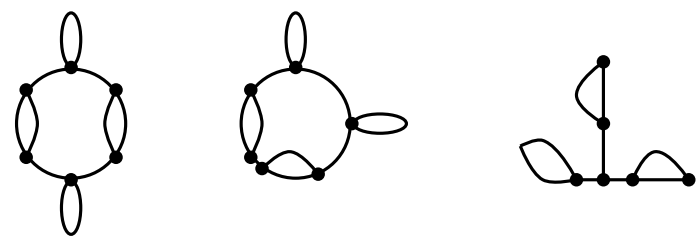

Figure 1: Two necklaces of type $(2,3)$ and a cactus with six vertices.

A bridge is an edge whose deletion increases the number of connected components. A bar-amalgamation of two disjoint graphs $H$ and $G$ is obtained by running an edge between a vertex of $G$ and a vertex of $H$. A cactus-free graph is inductively defined as follows: 1. Every 2-edge connected graph that is not a simple cycle is cactus-free. 2. The baramalgamation of two cactus-free graphs is cactus-free. The intuitive idea of a cactus-free graph $G$ is that when all the bridges are deleted from $G$, none of the components of the resulting graph is a simple cycle or an isolated vertex.

Let $G$ be a graph with minimum degree at least three. A frame of $G$ is obtained recursively by (1) for every vertex of degree four incident to a loop, deleting the loop and contracting one of the remaining incident edges, and (2) for every pair of vertices both of degree three and joined by two edges, contracting the three edges incident to one of them. A $C F$-graph is the frame of a cactus-free graph.
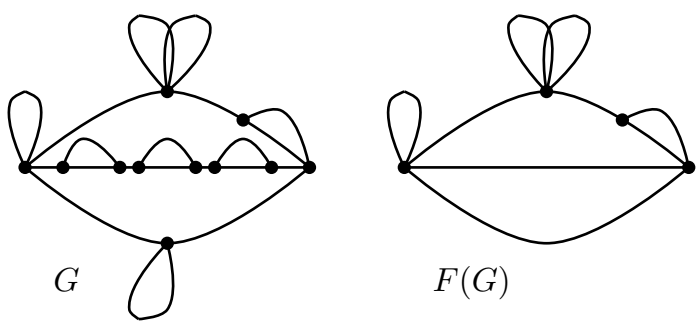

Figure 2: A graph $G$ and it's frame. 
Figure 1 gives an example of a graph $G$ and its frame $F(G)$.

In other words, a CF-graph can also be defined as a graph that does not contain the structures of Figure 1.

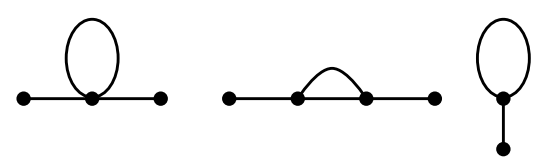

Figure 3: Three forbidden structures.

Note that Cacti and Necklaces $N_{r, s}(r, s \geqslant 1)$ are not CF-graphs, the average genus of each of the two graphs is bounded by 1 (see [4, 15], for details). In [3], J. Chen and J.L. Gross proved that a 2-connected simple graph with at least $9^{k}$ edges has average genus at least $\frac{k+1}{2}$. In other words, we have:

Theorem 1.1. (See [3], Theorem 4.3) Let $G$ be a 2-connected simple graph with minimum degree at least 3 , then the average genus $\gamma_{\text {avg }}(G)$ is larger than $c \log (\beta(G))$ for some constant $c>0$.

Note that each simple graph is a CF-graph. In [7], Chen improved this theorem as follows:

Theorem 1.2. (See [7], Theorem 4.5) Let $G$ be a CF-graph with minimum degree at least 3 , Then the average genus $\gamma_{\text {avg }}(G)$ is larger than $c \log (\beta(G))$ for some constant $c>0$.

In [9], we obtained the following result for the maximum genus of a CF-graph.

Theorem 1.3. (See [9]) Let $G$ be a CF-graph with minimum degree at least 3. Then lower bounds on the maximum genus are given in Table 1 . The rows correspond to edgeconnectivity $k=1$ or $k \geqslant 2$, respectively. The same bounds hold for vertex-connectivity $k$ and for graphs of arbitrarily large Betti number.

Table 1:

\begin{tabular}{|c|c|}
\hline$k$ & $\gamma_{M}(G)$ \\
\hline$k=1$ & $\min \left\{\frac{\beta(G)+2}{4},\left\lfloor\frac{\beta(G)}{2}\right\rfloor\right\}$ \\
$k \geqslant 2$ & $\min \left\{\frac{\beta(G)+2}{3},\left\lfloor\frac{\beta(G)}{2}\right\rfloor\right\}$ \\
\hline
\end{tabular}

Based on the above result, we will show a lower bound for the average genus of a CF-graph which is a linear function of its Betti Number.

Theorem 1.4. Let $G$ be a CF-graph with minimum degree at least 3. Then lower bounds on the average genus are given in Table 2 . The rows correspond to edge-connectivity $k=1$ or $k \geqslant 2$, respectively. The same bounds hold for vertex-connectivity $k$ and for graphs of arbitrarily large Betti number. 
Table 2:

\begin{tabular}{|l|ccc|}
\hline Type & Pseudograph & Multigraph & Simple \\
\hline$k=1$ & $\frac{\beta(G)}{20}$ & $\frac{\beta(G)}{12}$ & $\frac{\beta(G)}{8}[8]$ \\
$k \geqslant 2$ & $\frac{\beta(G)}{15}$ & $\frac{\beta(G)}{9}$ & $\frac{\beta(G)}{6}[2]$ \\
\hline
\end{tabular}

\section{The joint tree method}

By a polygon with $r$ edges, we shall mean a 2-cell which has its circumference divided into $r$ arcs by $r$ vertices. In fact, a surface can be obtained by pairing the edges of a polygon and identifying the two edges in each pair. The following three operations $[17,19]$ on a cyclic string representing such a polygon do not change genus of such a surface.

Operation 1: $A a a^{-} \sim A$,

Operation 2: $A a b B a b \sim A c B c$,

Operation 3: $A B \sim\left\{(A a),\left(a^{-} B\right)\right\}$,

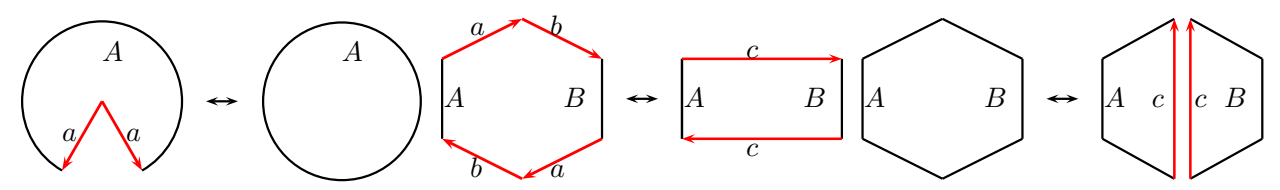

Figure 4: Operation 1, Operation 2 and Operation 3 (From left to right).

where $A$ and $B$ are all linear order of letters.

Property 2.1. (See [18], Principle 2 of P263) Let A, B, C and D be linear order of letters. Then $C x A B x^{-} D \sim D x B A x^{-} C$.

We have the following relation $[17,19]$.

Relation 1: $A a B b C a^{-} D b^{-} E \sim A D C B E a b a^{-} b^{-}$.

Proof. By Property 2.1,

$$
\begin{aligned}
A a B b C a^{-} D b^{-} E & \sim D b^{-} E a b C B a^{-} A=E a b C B a^{-} A D b^{-} \sim b a^{-} A D C B b^{-} E a \\
& \sim a^{-} b^{-} E A D C B a b=a b a^{-} b^{-} E A D C B \sim a b a^{-} b^{-} A D C B E .
\end{aligned}
$$

Relation 1 is also called handle normalization, In the above relation, $A, B$ and $C$ are permitted to be empty. By Relation 1, we can obtain the normal form of an orientable surface as one, and only one, of $O_{0}=a a^{-}, O_{m}=\prod_{i=1}^{m} a_{i} b_{i} a_{i}^{-} b_{i}^{-}(m>0)$.

The joint-tree approach [17] is an alternative to the Heffter-Edmonds algorithm for calculating the genus of the surface associated with a given rotation system. The rotation system is what combinatorializes the topological problem; a joint tree can be regarded as 
the combination of a spanning tree and a rotation system. Given a spanning tree $T$ and a rotation system $R$ of $G$, the associated joint tree, denoted by $G_{T}$ which is obtained by splitting each co-tree edge $e$ into two semi-edges $e$ and $e^{-}$. According to the rotation, all lettered semi-edges of $G_{T}$ form a polygon $P$ with $\beta(G)$ pairs of edges. Then, we apply Relation 1 and Operations 1,2 and 3 to normalize the polygon $P$ and get the genus of the embedding. Based on joint trees, the topological problem for determining embeddings of a graph is transformed into a combinatorial problem. For more details, we can also refer to $[22,23]$.

Example 2.2. Given a graph $G=(V, E), V=\left\{v_{1}, v_{2}, v_{3}, v_{4}\right\}, E=\{a, b, c, d, e, f\}, a, b$ and $d$ are edges on $T, c, e$ and $f$ are co-tree edges. The rotation system $R$ at each vertex is counterclockwise: $v_{1}(d e a), v_{2}(a f b), v_{3}(b e c), v_{4}(c f d)$. We travel along on $G_{T}$ according to the rotation system and obtain the polygon $c^{-} c f e f^{-} e^{-} \sim f e f^{-} e^{-}$, which is an embedding of $G$ into the torus (See Figure 2).



Figure 5: The graph $G$ and it's joint tree $G_{T}$.

Note that the polygon $P$ is described by a linear order of letters, we say these letters are elements of $P$.

Definition 2.3. Let $\Omega$ be a finite set. We call a polygon $P$ on $\Omega$ if every element of $P$ belongs to $\Omega$.

Definition 2.4. Let $P$ be the polygon obtained from a joint tree $G_{T}$. Assume that $P$ is a polygon on a finite set $\Omega$. Two elements $x, y \in \Omega$ are said to be interlaced on $P$ if it can be expressed as the form $P=A x B y C x^{-} D y^{-} E$, otherwise they are parallel on $P$.

Lemma 2.5. (See [17], Theorem 5.3) If any two elements are parallel on $P$, then there exists an element $x \in \Omega$ such that $P=A x x^{-} B$, where $A$ and $B$ are two linear orders of letters on $\Omega$.

Proof. Suppose $x \in \Omega$, and $P=A_{1} x_{1} B_{1} x_{1}^{-} C_{1}$ where $A_{1}, B_{1}$ and $C_{1}$ are three linear orders of letters on $\Omega$. If $B_{1}$ is empty, the theorem is true. Otherwise $B_{1}$ is nonempty, for any $x_{2} \in B_{1}$, on the basis of orientability and $x_{2}$ and $x_{1}$ parallel, the only possibility is $x_{2}^{-} \in B_{1}$. From the known condition, there is also a linear order $B_{2}$ on $\Omega$ such that $B_{1}=A_{2} x_{2} B_{2} x_{2}^{-} C_{2}$ where $A_{2}$ and $C_{2}$ are linear orders of letters on $B_{1}$. If $B_{2}$ is empty, the result follows. Otherwise $B_{2}$ is nonempty, and by the fact that the set of elements of $P$ is finite, we only repeat the above process finitely often and get the desired result. 
Lemma 2.6. (see [17], Theorem 5.4) Let $P$ be a polygon on $\Omega$. If $P \sim O_{k}(k \geqslant 1)$, then there exist two elements $x, y \in \Omega$ that are interlaced.

Proof. By contradiction, any elements of $P$ on $\Omega$ are parallel. By Lemma 2.1, we know that there exists an element $x \in \Omega$ such that $P=A x x^{-} B$, where $A$ and $B$ are linear orders of letters on $\Omega$. By Operation $1, P=A x x^{-} B \sim A B$. Since any elements of $A B$ are parallel too, by lemma 2.5, there exists an element $y \in \Omega$ such that $A B=C y y^{-} D$, where $C$ and $D$ are linear orders of letters on $\Omega$. By applying Operation 1 again, we have $A B=C y y^{-} D \sim C D$. Since the elements of $P$ is finite, at last we have $P \sim O_{0}$. This contradicts $P \sim O_{k}(k \geqslant 1)$.

Lemma 2.7. Let $P$ be a polygon on $\Omega$. If $P=A B C \sim O_{k}, P_{1}=x y A x^{-} B y^{-} C \sim O_{l}$ and $P_{2}=y x A x^{-} B y^{-} C \sim O_{n}$, then $l \geqslant k+1$ or $n \geqslant k+1$.

Proof. We prove the lemma by induction on number $k$. If $k=0$, by Relation $1, P_{1} \sim$ $B A C x y x^{-} y^{-} \sim O_{l}$. Since $l \geqslant 1$, it's true in this case. Now we suppose the result is true for $k=m \geqslant 1$. If we prove the theorem for $k=m+1$, then we complete the proof. Since $P=A B C \sim O_{k}(k \geqslant 1)$, by Lemma 2.6, there exist two elements $a, b \in \Omega$ are interlaced, i.e., $P=A_{1} a B_{1} b C_{1} a^{-} D_{1} b^{-} E_{1}$ where $A_{1}, B_{1}, C_{1}, D_{1}$, and $E_{1}$ are linear orders of letters on $\Omega$. So we can denote $P_{1}=x y A^{1} a B^{1} b C^{1} a^{-} D^{1} b^{-} E^{1}$ and $P_{2}=y x A^{1} a B^{1} b C^{1} a^{-} D^{1} b^{-} E^{1}$ where $A^{1}, B^{1}, C^{1}, D^{1}$, and $E^{1}$ are linear orders of letters on $\Omega \cup\left\{x^{-}, y^{-}\right\}$. By Relation 1 , we have

$$
\begin{aligned}
P & \sim A_{1} D_{1} C_{1} B_{1} E_{1} a b a^{-} b^{-}, \\
P_{1} & \sim x y A^{1} D^{1} C^{1} B^{1} E^{1} a b a^{-} b^{-}, \\
P_{2} & \sim y x A^{1} D^{1} C^{1} B^{1} E^{1} a b a^{-} b^{-} .
\end{aligned}
$$

If we denote $P^{\prime}=A_{1} D_{1} C_{1} B_{1} E_{1}=A^{\prime} B^{\prime} C^{\prime}$, two forms of $P_{1}^{\prime}$ and $P_{2}^{\prime}$ are discussed.

1. Case 1: $P_{1}^{\prime}=x y A^{1} D^{1} C^{1} B^{1} E^{1}=x y A^{\prime} x^{-} B^{\prime} y^{-} C^{\prime}$ and $P_{2}^{\prime}=y x A^{1} D^{1} C^{1} B^{1} E^{1}=$ $y x A^{\prime} x^{-} B^{\prime} y^{-} C^{\prime}$

2. Case 2: $P_{1}^{\prime}=x y A^{\prime} y^{-} B^{\prime} x^{-} C^{\prime}$ and $P_{2}^{\prime}=y x A^{\prime} y^{-} B^{\prime} x^{-} C^{\prime}$.

By symmetry, we need only to discuss case 1 . Since $P^{\prime} \sim O_{m}, P_{1}^{\prime} \sim O_{l-1}$ and $P_{2}^{\prime} \sim O_{n-1}$, by induction hypothesis, we have $l-1 \geqslant m$ or $n-1 \geqslant m$. So we get $P \sim O_{m+1}, P_{1} \sim O_{l}$ and $P_{2} \sim O_{n}$ where $l \geqslant m+1$ or $n \geqslant m+1$.

\section{The technique of vertex-splitting for a graph}

In this section, a special form of vertex-splitting of [16] is generalized.

Definition 3.1. Suppose the graph $G=(V, E)$ is simple. Let $u$ be a vertex of $G$ of valence $d(u)=d+1>3$ and $v, v_{1}, v_{2}, \ldots, v_{d}$ be its neighbors. We denote the edge $u v_{i}$ by $e_{i}$, for $i=1,2, \ldots, d$, and the edge $u v$ by $f$. The graph $G_{i_{1}, i_{2}, \ldots, i_{k}}$ is called a $k$-degree proper splitting of $G$ at $u$ if it can be obtained from $G-u$ by adjoining $v, v_{i_{1}}, v_{i_{2}}, \ldots, v_{i_{k}}$ to a new vertex $x$, adjoining all the other ex-neighbors of $u$ to a new vertex $y\left(i_{l} \in\{1,2, \ldots, k\}\right.$, for $l=1,2, \ldots, k$ and $d>k \geqslant 1$ ), and finally adjoining $x$ and $y$. 
The new vertex $x$ is $(k+2)$-valent for each $G_{i_{1}, i_{2}, \ldots, i_{k}}$ and the new vertex $y$ is $(d-k+1)$ valent. Let $\Lambda$ be the set of all graphs $G_{i_{1}, i_{2}, \ldots, i_{k}}$, then the number of elements in $\Lambda$ is $\left(\begin{array}{l}d \\ k\end{array}\right)$. It is obvious that each graph $G_{i_{1}, i_{2}, \ldots, i_{k}}$ has the same the Betti number as that of $G$, and they can contract the new edge $x y$ to get the graph $G$. Figure 6

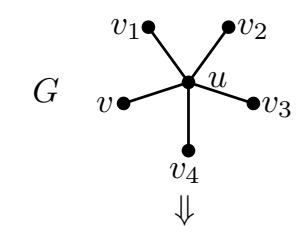

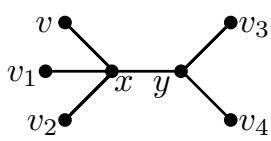

$G_{12}$

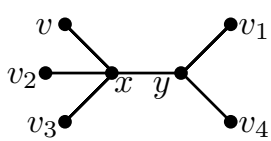

$G_{23}$

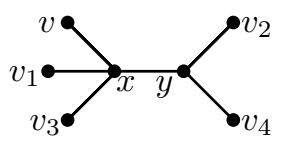

$G_{13}$



$G_{24}$



$G_{14}$

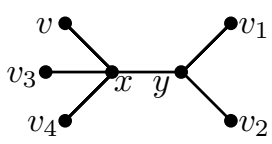

$G_{34}$

Figure 6: The 2-degree proper splitting of $G$ at $u$ with a designate neighbor $v$

gives an example of a 2-degree proper splitting of $G$ at $u$.

Suppose the rotation system $R$ of $G$ at vertex $u$ is

$$
\text { u. } e_{i_{1}} e_{i_{2}} \ldots e_{i_{d}} f
$$

where $i_{j} \in\{1,2, \ldots, d\}$, for $j=1,2, \ldots, d$ and $f$ is the edge $u v$. Let $R_{i_{1}, i_{2}, \ldots, i_{k}}$ be the rotation system of the graph $G_{i_{1}, i_{2}, \ldots, i_{k}}$ with rotations

$$
\text { x. } f e_{i_{1}} \ldots e_{i_{k}} e \text { and } y . e e_{i_{k+1}} \ldots e_{i_{d}}
$$

and all other vertex rotations as in $R$. $e$ is the new edge in $G_{i_{1}, i_{2}, \ldots, i_{k}}$ that connects the new vertex $x$ and $y$. Let $R_{i_{d-k+1}, \ldots, i_{d}}$ be the rotation system of the graph $G_{i_{d-k+1}, \ldots, i_{d}}$ with rotations

$$
\text { x. fee } e_{i_{d-k+1}} e_{i_{d-k+2}} \ldots e_{i_{d}} \text { and } y . e e_{i_{1}} \ldots e_{i_{d-k}}
$$

and all other vertex rotations as in $R$. Similarly $R_{i_{j}, \ldots, i_{d}, i_{1}, \ldots, i_{j+k-d-1}}$ be the rotation system of $G_{i_{j}, \ldots, i_{d}, i_{1}, \ldots, i_{j+k-d-1}}$, for $j=d-k+2, \ldots, d$. with rotations

$$
\text { x. } e_{i_{j}} \ldots e_{i_{d}} f e_{i_{1}} \ldots e_{i_{k+j-d-1}} e \quad \text { and } y . e e_{i_{k+j-d}} \ldots e_{i_{j-1}}
$$

and all other vertex rotations as in $R$.

Definition 3.2. The rotation systems $R_{i_{1}, i_{2}, \ldots, i_{k}}, R_{i_{d-k+1}, \ldots, i_{d}}$ and $R_{i_{j}, \ldots, i_{d}, i_{1}, \ldots, i_{j+k-d-1}}$, for $j=d-k+2, \ldots, d$, are said to be obtained by a $k$-degree proper splitting at the vertex $u$ in the rotation system $R$ with the designated neighbor $v$ 
Note that the rotation system $R$ can be obtained by contracting the rotation system $R_{i_{1}, i_{2}, \ldots, i_{k}}, R_{i_{d-k+1}, \ldots, i_{d}}$ or $R_{i_{j}, \ldots, i_{d}, i_{1}, \ldots, i_{j+k-d-1}}$, for $j=d-k+2, \ldots, d$, on the edge $e$. Furthermore we have:

Lemma 3.3. Let $G$ be a connected simple graph with a vertex $u$ of valence $d+1(d>3)$ and a neighbor $v$. Let $R$ be a rotation system of $G$. Then there are exactly $k+1$ systems of the $k$-degree proper splittings of $G$ at $u$ with designated neighbor $v$ that are $k$-degree proper splittings of $R$. Moreover, every rotation system of a $k$-degree proper splitting of $G$ is uniquely contractible on the edge xy to a rotation system of $G$.

Proof. Suppose the rotation system $R$ at vertex $u$ is

$$
\text { u. } e_{i_{1}} e_{i_{2}} \ldots e_{i_{d}} f
$$

where $i_{j} \in\{1,2, \ldots, d\}$, for $j=1,2, \ldots, d$ and $f$ is the edge $u v$. By the definition, $R$ can be obtained only by contracting the edge $e$ in the rotation systems $R_{i_{1}, i_{2}, \ldots, i_{k}}, R_{i_{d-k+1}, \ldots, i_{d}}$ or $R_{i_{j}, \ldots, i_{d}, i_{1}, \ldots, i_{j+k-d-1}}$, for $j=d-k+2, \ldots, d$, which are defined above. Furthermore, each of them is uniquely contractible on $e$ to the rotation system $R$.

In the genus polynomial $g_{G}(x)=\sum_{k \geqslant 0} g_{k} x^{k}$ of $G$, the coefficient of $x^{k}$ is the number of distinct embeddings of the graph $G$ on the oriented surface of genus $k$. Note that when a graph $G$ is non-simple, we can subdivide the multiple edges and loops of $G$ and obtain a simple graph. Since they have the same genus polynomial, by Lemma 3.3, we have:

Lemma 3.4. Let $G$ be a connected graph with a vertex $u$ of valence $d+1(d \geqslant 3)$, and let $G_{i_{1}, i_{2}, \ldots, i_{k}}\left(i_{j} \in\{1,2, \ldots, d\}\right)$ be graphs obtained by $k$-degree properly splitting at vertex $u$, and $\Lambda$ be the sets of all the graphs $G_{i_{1}, i_{2}, \ldots, i_{k}}$. Then we have

$$
g_{G}(x)=\frac{1}{k+1} \sum_{G_{i_{1}, i_{2}, \ldots, i_{k}} \in \Lambda} g_{G_{i_{1}, i_{2}, \ldots, i_{k}}}(x) .
$$

It is routine to check the following corollary by the definition of average genus and lemma 3.4.

Corollary 3.5. Let $G$ be a connected graph with a vertex $u$ of valence $d+1(d \geqslant 3)$, and let $G_{i_{1}, i_{2}, \ldots, i_{k}}\left(i_{j} \in\{1,2, \ldots, d\}\right)$ be graphs obtained by $k$-degree properly splitting at vertex $u$, and $\Lambda$ be the sets of all the graphs $G_{i_{1}, i_{2}, \ldots, i_{k}}$. Then we have $\gamma_{\text {avg }}(G)=$ $\frac{1}{\left(\begin{array}{c}d \\ k\end{array}\right)} \sum_{G_{i_{1}, i_{2}, \ldots, i_{k}} \in \Lambda} \gamma_{\text {avg }}\left(G_{i_{1}, i_{2}, \ldots, i_{k}}\right)$.

\section{Lower bound for the average genus of a graph}

In [6] it was shown that the average genus of a 3-regular graph is at least half its maximum genus, we will obtain a more general result in this section. Let $G^{\prime}$ be a subgraph of a 
graph $G$ and $R$ be a rotation system on $G$. The induced rotation system $R^{\prime}$ on $G^{\prime}$ is obtained by deleting all edges of $G-G^{\prime}$ from the rotation system $R$. Let $\Gamma$ and $\Gamma^{\prime}$ be the sets of rotation systems on $G$ and $G^{\prime}$ respectively. We denote $\Gamma_{R^{\prime}}$ the set of all rotation systems on $G$ that induce rotation system $R^{\prime}$ on $G^{\prime}$. The following Lemma is obtained from $[6]$.

Lemma 4.1. (see [6]) Let $G^{\prime}$ be a subgraph of a graph $G$. Then the set $\Gamma$ of all rotation systems on $G$ is a disjoint union of the sets $\Gamma_{R^{\prime}}$, taken over all rotation systems $R^{\prime}$ on $G^{\prime}$. Moreover, $|\Gamma|=\left|\Gamma^{\prime}\right| \cdot\left|\Gamma_{R^{\prime}}\right|$, for any rotation system $R^{\prime}$ on the graph $G^{\prime}$.

Lemma 4.2. (see [6]) Let $G$ be a graph of maximum genus greater than 0 . Then there exist a pair of adjacent edges $\{e, f\}$ such that the graph $G^{\prime}=G-e-f$ is a connected spanning subgraph of $G$ and $\gamma_{M}(G)=\gamma_{M}\left(G^{\prime}\right)+1$.

Now we have the following theorem:

Theorem 4.3. Let $G$ be a graph of maximum degree at most $d$. Then $\gamma_{\mathrm{avg}}(G) \geqslant \frac{\gamma_{M}(G)}{d-1}$.

Proof. We prove the Theorem by induction on the number $\gamma_{M}(G)$. If $\gamma_{M}(G)=0$, by the definition of average genus, we know that the average genus of $G$ is also 0 . Now we suppose that the graph $G$ has maximum genus not less than 1. By Lemma 4.2, there exist a pair of adjacent edges $\{e, f\}$ in $G$ such that the graph $G^{\prime}=G-e-f$ is a connected spanning subgraph of $G$ and $\gamma_{M}(G)=\gamma_{M}\left(G^{\prime}\right)+1$. Suppose $e$ and $f$ are incident with a common vertex $v$. Without loss of generality, we let $e=u v$ and $f=v w$ where $u, v$ and $w$ are distinct vertices of $G$ (when $G$ is a non-simple graph, we can subdivide the loops and multiple edges of $G$ ). It is evident that the maximum degree of $G^{\prime}$ is also at most $d$. By our inductive hypothesis, the average genus of $G^{\prime}$ is not less than $\frac{\gamma_{M}\left(G^{\prime}\right)}{d-1}$. i.e.,

$$
\gamma_{\mathrm{avg}}\left(G^{\prime}\right) \geqslant \frac{\gamma_{M}\left(G^{\prime}\right)}{d-1}=\frac{\gamma_{M}(G)-1}{d-1}=\frac{\gamma_{M}(G)}{d-1}-\frac{1}{d-1} .
$$

Let $R$ be a rotation system on $G$ and $R^{\prime}$ be a rotation system on $G^{\prime}$. Let $\Gamma$ and $\Gamma^{\prime}$ be the sets of rotation system on $G$ and $G^{\prime}$ respectively. We denote $\Gamma_{R^{\prime}}$ the set of all rotation systems on $G$ that induce rotation system $R^{\prime}$ on $G^{\prime}$. It is easy to see that $\left|\Gamma_{R^{\prime}}\right|=\left(d_{G}(v)-1\right)\left(d_{G}(v)-2\right)\left(d_{G}(u)-1\right)\left(d_{G}(w)-1\right)$. Note that the genus polynomial $g_{G}(x)$ is independent of the choice of the spanning tree $T$. To the rotation system $R^{\prime}$ on $G^{\prime}$, by joint-tree method, we can obtained a joint tree $G_{T}^{\prime}$ and a polygon $P^{\prime}$. Similarly, To the rotation system $R$ of $\Gamma_{R^{\prime}}$, we also can get a joint tree $G_{T}$ and a polygon $P$. By the relation between $R^{\prime}$ and $R$, if we denote $P^{\prime}=A B C D$, we can express $P=e A f B e^{-} C f^{-} D$ or $P=f A e B e^{-} C f^{-} D$. It is easy to see that there are $\lambda=\left(d_{G}(v)-2\right)\left(d_{G}(u)-1\right)\left(d_{G}(w)-1\right)$ pairs of $\left\{e f B e^{-} C f^{-} D, f e B e^{-} C f^{-} D\right\}$ ( i.e, A is empty). By Lemma 2.7, for each pair $\left\{\right.$ ef $\left.B e^{-} C f^{-} D, f e B e^{-} C f^{-} D\right\}$ of polygon, one of the genus $\left\{e f B e^{-} C f^{-} D, f e B e^{-} C f^{-} D\right\}$ is greater than that of $B C D$ by one. Consequently, at least $\frac{\lambda}{\left|\Gamma_{R^{\prime}}\right|}=\frac{1}{d_{G}(v)-1}$ rotation systems in the set $\Gamma_{R^{\prime}}$ have genus at least $\gamma\left(R^{\prime}\right)+1$ and no rotation system in the set $\Gamma_{R^{\prime}}$ has genus less than $\gamma\left(R^{\prime}\right)$. According to Lemma 4.1 and Inequality (1), we have 


$$
\begin{aligned}
\gamma_{\mathrm{avg}}(G)= & \frac{\sum_{R \in \Gamma} \gamma(R)}{|\Gamma|}=\frac{\sum_{R^{\prime} \in \Gamma^{\prime}} \sum_{R \in \Gamma_{R^{\prime}}} \gamma(R)}{|\Gamma|} \geqslant \frac{\sum_{R^{\prime} \in \Gamma^{\prime}}\left(\left|\Gamma_{R^{\prime}}\right| \gamma\left(R^{\prime}\right)+\frac{\left|\Gamma_{R^{\prime}}\right|}{d_{G}(v)-1}\right)}{|\Gamma|} \\
= & \frac{\sum_{R^{\prime} \in \Gamma^{\prime}}\left(\gamma\left(R^{\prime}\right)+\frac{1}{d_{G}(v)-1}\right)}{\left|\Gamma^{\prime}\right|}=\gamma_{\mathrm{avg}}\left(G^{\prime}\right)+\frac{1}{d_{G}(v)-1} \geqslant \gamma_{\mathrm{avg}}\left(G^{\prime}\right)+\frac{1}{d-1} \\
\geqslant & \frac{\gamma_{M}(G)}{d-1} .
\end{aligned}
$$

\section{The proof of Theorem 1.4}

Proof. Let the number of vertices with maximum degree $\triangle(G)$ is $n$. We prove the theorem by induction on the number $n+\triangle(G)$.

Case 1: $G$ is a multigraph.

Subcase a: $\kappa_{1}(G)=1$. If the maximum degree $\triangle(G)$ of $G$ is less than 5 , by Theorem 1.3 and Theorem 4.3, we have

$$
\gamma_{\text {avg }}(G) \geqslant \min \left\{\frac{\beta(G)+2}{12}, \frac{\left\lfloor\frac{\beta(G)}{2}\right\rfloor}{3}\right\}=\left\{\begin{array}{ll}
\frac{1}{3}, & \beta(G)=3 \\
\frac{\beta(G)+2}{12}, & \beta(G) \geqslant 4
\end{array}>\frac{\beta(G)}{12} .\right.
$$

Otherwise $\triangle(G) \geqslant 5$, the following two different cases are discussed. (In this case, we have $\beta(G) \geqslant 5)$.

(1). $\triangle(G)=5$. Let $u$ be a vertex of degree $\triangle(G)$. Then the edge set $E(u)=\{u v$ : $u v \in E(G)\}$ is isomorphic to one of the seven cases in Figure 7 .

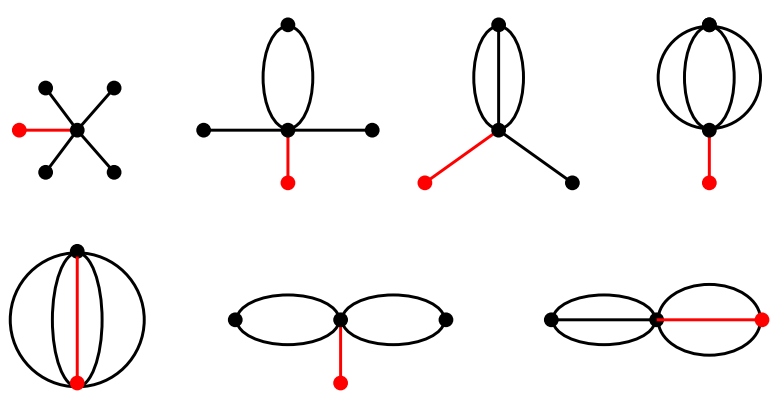

Figure 7: Seven cases.

To each case, we construct four graphs $G_{2}, G_{3}, G_{4}$ and $G_{5}$ by a 1-degree proper splitting at the vertex $u$ with a designated neighbor such that the red edge incident with. It is a routine task to check that each graph $G_{i}$, for $i=2,3,4,5$, is a CF-graph and the minimum 
degree of $G_{i}$ is at least 3 . Note that each graph $G_{i}$, for $i=2,3,4,5$, has the same Betti number as that of $G$. By our inductive hypothesis, all the graphs $G_{2}, G_{3}, G_{4}$, and $G_{5}$ have average genus at least $\frac{\beta(G)}{12}$. By Corollary 3.5,

$$
\gamma_{\text {avg }}(G)=\frac{1}{4} \sum_{i=2}^{5} \gamma_{\text {avg }}\left(G_{i}\right) \geqslant \frac{1}{4} \sum_{i=2}^{5} \frac{\beta(G)}{12}=\frac{\beta(G)}{12} .
$$

(2). $\triangle(G) \geqslant 6$. Let $u$ be a vertex of degree $\triangle(G)$. We construct $(\underset{2}{\Delta(G)-1})$ graphs $G_{1}, G_{2}, \ldots, G_{(\triangle(G)-1)}$ by a 2 -degree proper splitting at the vertex $u$ with a designated neighbor (any vertex adjacent to $u$ ). Let $\Lambda$ be the set of graphs $G_{1}, G_{2}, \ldots, G_{\left(\begin{array}{c}\Delta(G)-1 \\ 2\end{array}\right)}$. Since each graph $G_{i}$, for $i=1,2, \ldots,\left(\begin{array}{c}\triangle(G)-1 \\ 2\end{array}\right)$, is a CF-graph and the minimum degree of $G_{i}$ is at least 3 , by our inductive hypothesis, all the graphs $G_{1}, G_{2}, \ldots, G_{(\underset{2}{\Delta(G)-1})}$ have average genus at least $\frac{\beta(G)}{12}$. By Corollary 3.5,

$$
\gamma_{\text {avg }}(G)=\frac{1}{\left(\begin{array}{c}
(G)-1 \\
2
\end{array}\right)} \sum_{G \in \Lambda} \gamma_{a v g}(G) \geqslant \frac{\beta(G)}{12} .
$$

Subcase b: $\kappa_{1}(G) \geqslant 2$. In this case have a similar discussion as in subcase a.

Case 2: $G$ is a pseudograph.

Subcase 1: $\kappa_{1}(G)=1$. If the maximum degree $\triangle(G)$ of $G$ is less that 7 , by Theorem 1.3 and Theorem 4.3, we know that the theorem is true. Otherwise $\triangle(G) \geqslant 7$, the following two different cases are discussed.

(1). $\triangle(G)=7$. Let $u$ be a vertex of degree $\triangle(G)$. Then the edge set $E(u)=\{u v$ : $u v \in E(G)\}$ is isomorphic to one of twenty six cases in Figure 8 .

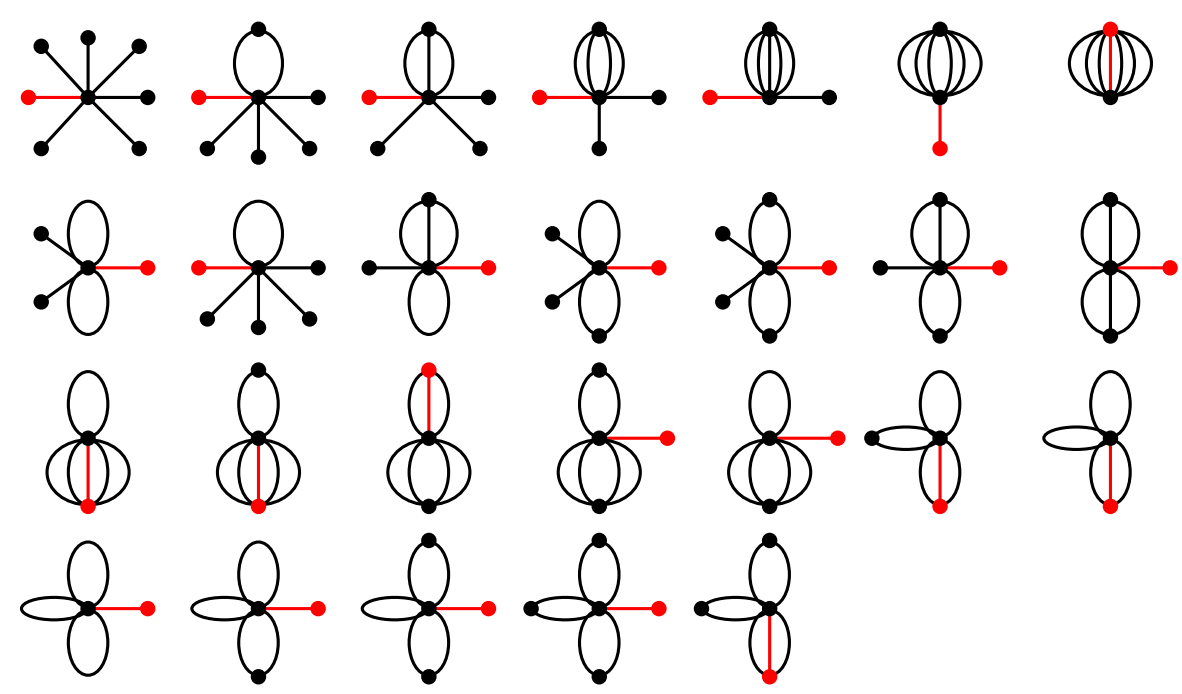

Figure 8: Twenty six cases.

To each case, we construct six graphs $G_{2}, G_{3}, \ldots, G_{7}$ by a 1-degree proper splitting at the vertex $u$ with a designated neighbor which the red edge is incident with. It is a routine 
task to check that each graph $G_{i}$, for $i=2,3, \ldots, 7$, is a CF-graph and the minimum degree of $G_{i}$ is at least 3 . By inductive hypothesis, all the graphs $G_{2}, G_{3}, \ldots, G_{7}$ have average genus at least $\frac{\beta(G)}{20}$. Then from Corollary 3.5,

$$
\gamma_{a v g}(G)=\frac{1}{6} \sum_{i=2}^{7} \gamma_{a v g}\left(G_{i}\right) \geqslant \frac{1}{6} \sum_{i=2}^{7} \frac{\beta(G)}{20}=\frac{\beta(G)}{20}
$$

(2). $\triangle(G) \geqslant 8$. Let $u$ be a vertex of degree $\triangle(G)$. We construct $\left(\begin{array}{c}\triangle(G)-1 \\ 3\end{array}\right)$ graphs $G_{1}, G_{2}, \ldots, G_{(\underset{3}{\triangle(G)-1})}$ by a 3 -degree proper splitting at the vertex $u$ with a designated neighbor (any vertex adjacent to $u$ ). Let $\Lambda$ be the set of graphs $G_{1}, G_{2}, \ldots, G_{(\triangle(G)-1)}$. Since each graph $G_{i}$, for $i=1,2, \ldots,\left(\begin{array}{c}\triangle(G)-1 \\ 3\end{array}\right)$, is a CF-graph and the minimum degree of $G_{i}$ is at least 3 , by our inductive hypothesis, all the graphs $G_{i}$ have average genus at least $\frac{\beta(G)}{20}$. By Corollary 3.5,

$$
\gamma_{a v g}(G)=\frac{1}{\left(\begin{array}{c}
\triangle(G)-1 \\
3
\end{array}\right)} \sum_{G \in \Lambda} \gamma_{a v g}(G) \geqslant \frac{\beta(G)}{20} .
$$

Subcase 2: $\kappa_{1}(G) \geqslant 2$. In this case have a similar discussion as in subcase 1 .

\section{Some additional results}

In [3] it was proved that the distribution of average genus of simple graphs is sparse in the real line $R$. By Theorem 1.4, we know that simple graphs can be replaced by CF-graphs.

Theorem 6.1. Let $r$ be a positive real number, then only finitely many real numbers less than $r$ are possible values of average genus for CF-graphs.

Theorem 6.2. (see [15]) The average genus of a graph is not less than the average genus of any of its subgraphs.

Let $e \in E(G)$, if we insert two vertices $u$ and $v$ and double the edge between them, we say we attach an open ear to the interior of $e$. Similarly, if the vertices $u=v$, then we say we attach $a$ closed ear to the interior of $e$. The two vertices $u$ and $v$ are called the ends of the ear. We say $r$ open ears and $s$ closed ears are attached serially to the edge $e$, if all ends of the ears are distinct. A sequence $G_{1}, G_{2}, G_{3}, \ldots$, of graphs is called strictly monotone sequence if no pair of graphs in the sequence are homeomorphic and each $G_{i}$ is homeomorphic to a subgraph of $G_{i+1}$ for all $i \geqslant 1$. In [4] it was proved that the values of the average genus for 2-connected graphs have limit points. Note that the average genus for bar-amalgamation of a cactus and the graph $G$ equals to the average genus of $G$. By Theorem 1.4, the limit points for average genus may not be bounded in 2-connected graphs. We have the following result as a generalization.

Theorem 6.3. Let $G_{1}, G_{2}, G_{3}, \ldots$, be a strictly monotone sequence of connected graphs such that the values of the average genus of the graphs approach a finite limit point. Then there exists an index $N$ such that all but a finite number of graphs in the sequence can be obtained by attaching ears serially or by bar-amalgamation of a cactus to $G_{N}$. 
Proof. Suppose the values of the average genus of the graphs approach a finite limit point $L$, i.e.,

$$
\lim _{i \rightarrow \infty} \gamma_{a v g}\left(G_{i}\right)=\lim _{\beta\left(G_{i}\right) \rightarrow \infty} \gamma_{a v g}\left(G_{i}\right)=L
$$

By Theorem 6.2, we have $\gamma_{a v g}\left(G_{i}\right) \geqslant \gamma_{a v g}\left(G_{i-1}\right)(i \geqslant 2)$. By Theorem $1.4, G_{n}(n$ is sufficiently large) must not be a CF-graph. Since $G_{1}, G_{2}, G_{3}, \ldots$, be a strictly monotone sequence of connected graphs, there must exist an index $N$ such that each graph $G_{i}$ can be obtained by attaching ears serially or by bar-amalgamation of a cactus to $G_{N}$

The authors of [5] discussed a Kuratowski type theorem for average genus of graphs. They obtained the structure of average genus less than 1 with the help of computer, and also posed a problem to characterize the structure of average genus less than a fixed constant $c$ systematically.

Theorem 6.4. (see [5]) A cactus-free graph $G$ has average genus less than 1 if and only if either $G$ is a necklace or homeomorphic to one of finitely many exceptions.

Actually, the above theorem can also be extended to the general case. We have the following generalized Kuratowski type theorem of [5].

Theorem 6.5. A cactus-free graph $G$ has average genus less than $c$ if and only if either $G$ is obtained by attaching ears or $G$ is homeomorphic to one of finitely many exceptions.

Proof. Let $G$ be a graph whose average genus less than $c$. If $G$ is a CF-graph, by Theorem 1.4, there are finitely many $\mathrm{CF}$-graphs with average genus less than $c$, then the theorem is true. Otherwise, by Theorem 1.4, it can be obtained by attaching ears to one of finitely many CF-graphs.

\section{Acknowledgements}

I am grateful to the anonymous referee for pointing out a simple proof of Relation 1 by using Property 2.1 and Professor Tommy Jensen for his patience and detail comments on a former version of the paper. Thanks are also given to Professor Yanpei Liu for his guidance to topological graph theory. The work was supported by the National Science Foundation of China under Grant No. 10901048.

\section{References}

[1] D. Archdeacon, Calculations on the average genus and genus distribution of graphs, Congr. Numer. 67 (1988) 114-124.

[2] D. Archdeacon, J. Chen, Y. Huang, S.P. Kanchi, D. Li, Y.P. Liu, R. Nedela and M. Skoviera, Maximum Genus, Connectivity, and Nebesky's Theorem. http://www . emba.uvm. edu/ archdeac/papers/papers.html.

[3] J. Chen and J.L. Gross, Limit points for average genus (I) 3-Connected and 2Connected Simplicial Graphs, J. Combin. Theory Ser. B 55 (1992) 83-103. 
[4] J. Chen and J.L. Gross, Limit points for average genus (II) 2-Connected nonsimplicial graphs, J. Combin. Theory Ser. B 56 (1992) 108-129.

[5] J. Chen and J.L. Gross, Kuratowski-type theorems for average genus, J. Combin. Theory Ser. B 57 (1993) 100-121.

[6] J. Chen, J.L. Gross and R.G. Rieper, Lower Bounds for the Average Genus, J. Graph Theory 19 (1995) 281-296.

[7] J. Chen, A Linear-Time Algorithm for Isomorphism of Graphs of Bounded Average Genus, SIAM J. Discrete Math. 7 (4) (1994) 614-631.

[8] J. Chen, S. P. Kanchi and J.L. Gross, A tight lower bound on the maximum genus of a simplicial graph, Discrete Math. 156 (1-3) (1996) 83-102.

[9] Y. Chen and Y. Liu, A note on the lower bounds for the maximum genus. Util. Math. 73 (2007) 23-31.

[10] Y. Chen, A note on a conjecture of S. Stahl, Canad. J. Math. 60 (4) (2008) 958-959.

[11] Y. Chen and Y. Liu, On a conjecture of S. Stahl, Canad. J. Math. 62 (5) (2010) 1058-1059.

[12] Y. Chen, Y. Liu and Q. Zhou, On the average crosscap number of a graph. Preprint

[13] Y. Chen and Y. Liu, On the average crosscap number II: Bounds for a graph, Sci. China Ser. A 50 (2007) 292-304.

[14] J.L. Gross and T. Tucker, Topological Graph Theory, Wiley, New York, 1987.

[15] J.L. Gross, E.W. Klein and R.G. Rieper, On the average genus of a graph, Graphs and Combin. 9 (1993) 153-162.

[16] J.L. Gross, Genus distribution of graphs under surgery: adding edges and splitting vertices, New York J. Math. 16 (2010) 161-178.

[17] Y. Liu, Advances in Combinatorial Maps (In Chinese), Northern JiaoTong Unversity Press, Beijing, 2003.

[18] Y. Liu, Theory of Polyhedra, Science Press, Beijing, 2008.

[19] G. Ringel, Map Color Theory, Springer, Berlin, 1974.

[20] S. Stahl, An upper bound for the average number regions, J. Combin. Theory Ser. B 52 (1991) 191-218.

[21] S. Stahl, On the average genus of the random graph, J. Graph Theory 20 (1995) $1-18$.

[22] L. Wan and Y. Liu, Orientable embedding genus distribution for certain types of graphs, J. Combin. Theory Ser. B 98 (1) (2008) 19-32.

[23] Y. Yang and Y. Liu, Classification of $(p, q, n)$-dipoles on nonorientable surfaces, Electron. J. Combin. 17 (2010) \#N12. 